\title{
A PROCEDURE FOR THE MOTION OF PARTICLE- ENCAPSULATED DROPLETS IN MICROCHANNELS
}

\author{
Y. F. Yap ${ }^{1}$, J. C. Chai ${ }^{1}$, T. N. Wong', N. T. Nguyen ${ }^{1}$, \\ K. C. Toh', H. Y. Zhang' ${ }^{2}$, and L. Yobas ${ }^{2}$ \\ ${ }^{1}$ School of Mechanical \& Aerospace Engineering, Nanyang Technological \\ University, Singapore \\ ${ }^{2}$ Institute of Microelectronics, Singapore
}

A fixed-grid approach for modeling the motion of a particle-encapsulated droplet carried by a pressure-driven immiscible carrier fluid in a microchannel is presented. Three phases (the carrier fluid, the droplet, and the particle) and two different moving boundaries (the dropletcarrier fluid and droplet-particle interfaces) are involved. This is a moving-boundaries problem with the motion of the three phases strongly coupled. In the present article, the particle is assumed to be a fluid of high viscosity and constrained to move with rigid body motion. A combined formulation using one set of governing equations to treat the three phases is employed. The droplet-carrier fluid interface is represented and evolved using a level-set method with a mass-correction scheme. Surface tension is modeled using the continuum surface force model. An additional signed distance function is employed to define the droplet-particle interface. Its evolution is determined from the particle motion governed by the Newton-Euler equations. The governing equations are solved numerically using a finite-volume method on a fixed Cartesian grid. For demonstration purposes, the flows of particle-encapsulated droplets through a constricted microchannel and through a microchannel system are presented.

\section{INTRODUCTION}

Droplet-based or digital microfluidic has attracted much attention recently. One of the applications of microscopic droplets is to transport cargos on microfluidic platforms [1]. These cargos include but are not limited to cells and cellular organelles. For ease of discussion, these are referred collectively as particles in the present article, given their microscopic sizes. Particles encapsulated inside a droplet can be generated in specially designed microchannel configurations. Fisher and Lee [2] demonstrated cell encapsulation in a novel microfluidic device. Encapsulation of single cell and subcellular organelles into even smaller droplets separately on two different designs, i.e., a T-junction and a constricted microchannel, has been performed successfully [3]. Given the small size of the droplet (with encapsulated particle), a large number of droplets can be generated and manipulated in parallel in a single

Address correspondence to John C. Chai, Nanyang Technological University, School of Mechanical and Aerospace Engineering, Nanyang Avenue, Singapore 639798. E-mail: mckchai@ntu.edu.sg 


\begin{tabular}{|c|c|c|c|}
\hline \multicolumn{4}{|c|}{ NOMENCLATURE } \\
\hline$\vec{F}$ & resultant surface force & $\phi$ & signed distance function for $\mathrm{L}-\mathrm{S}$ \\
\hline$I$ & moment of inertia of the particle & & interface \\
\hline$\hat{k}$ & unit vector in the $z$ direction & $\delta$ & Dirac delta function \\
\hline$M_{c}$ & $\begin{array}{l}\text { the most current mass of the reference } \\
\text { phase }\end{array}$ & $\varepsilon$ & $\begin{array}{l}\text { a parameter proportional to the mesh } \\
\text { size }\end{array}$ \\
\hline$M_{d}$ & desired mass of the reference phase & $\theta$ & orientation of the particle \\
\hline$\hat{n}$ & unit outward normal of the $\mathrm{L}-\mathrm{S}$ & $\kappa$ & curvature \\
\hline & interface & $\xi, \psi, \psi^{\prime}$ & level-set functions \\
\hline $\bar{t}, t^{\prime}$ & pseudo-times & $\overline{\bar{\sigma}}$ & stress tensor \\
\hline$\vec{T}$ & torque & \\
\hline$\vec{u}$ & velocity vector & Subscrip & \\
\hline$\vec{x}$ & position vector & $d$ & droplet \\
\hline$\vec{x}_{p}$ & centroid of the particle & $c$ & carrier fluid \\
\hline$\Gamma_{L}$ & $\mathrm{~L}-\mathrm{L}$ interface & $p$ & particle \\
\hline$\Gamma_{p}$ & $\mathrm{~L}-\mathrm{S}$ interface & ref & reference phase \\
\hline
\end{tabular}

microfluidic device. The particles are transported in the form of encapsulations in the droplets carried by an immiscible carrier fluid in microchannels. The droplets provide the encapsulated particles both physical and chemical isolation. This is potentially useful for single-cell or single-organelle study in a controlled manner. Chemical analysis can be initiated on the encapsulated particle by fusing the droplet which contains the encapsulated particle with another droplet containing appropriate reagents in the transportation process.

When these droplets are transported in the carrier fluid, they undergo deformation. Under certain conditions, the particle may break out from their encapsulation and thus be contaminated by the carrier fluid. This is highly undesirable. Therefore a sound understanding of the motion of these droplets with the encapsulated particles in different microchannel geometries is essential to ensure reliable transportation.

Droplet transport in microchannels was presented by Yap et al. [4]. A level-set method [5] was used to capture the interface of the droplet. Yap et al. [6] also presented a method to model particle transport in microchannels. The method was used to model circular and elliptical particles.

This article presents a fixed-grid approach to model the motion of a droplet encapsulating a solid particle of finite size. The droplet together with its content (a solid particle) is driven by another immiscible carrier fluid in microchannels. The solid particle can be thought of as an encapsulated cell. As shown in Figure 1, encapsulation transport is a combination of the droplet transport [4] and the particle transport [6].

The remainder of the article is divided into three sections. In the following section, the theoretical model is first presented. This is followed by the results obtained and discussion. Finally, concluding remarks are given.

\section{PROBLEM DESCRIPTIONS}

Figure 2 shows two situations considered in this article. These are (1) a droplet containing a particle flowing in a microchannel with a constriction and (2) a droplet 


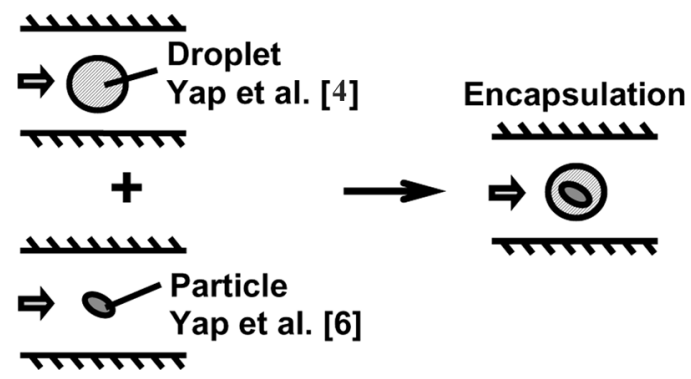

Figure 1. Encapsulation transport as combined droplet transport and particle transport.

containing a particle flowing in a microchannel system. Three phases, namely, the carrier fluid, the droplet, and the solid particle, are involved in these problems.

In Figure $2 a$, the centroid of the elliptical particle is located at $\left(x_{p}, y_{p}\right)$. The semimajor and semiminor axes of the elliptical particle are $a$ and $b$, respectively. A circular particle can be obtained by setting $a=b$. Initially, this particle is encapsulated in a circular droplet of diameter $d_{d}$ and centered at $\left(x_{d}, y_{d}\right)$. The carrier fluid

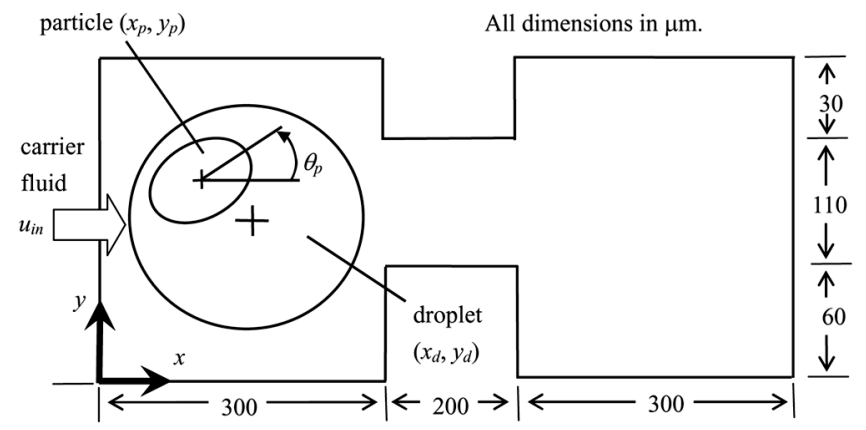

(a)

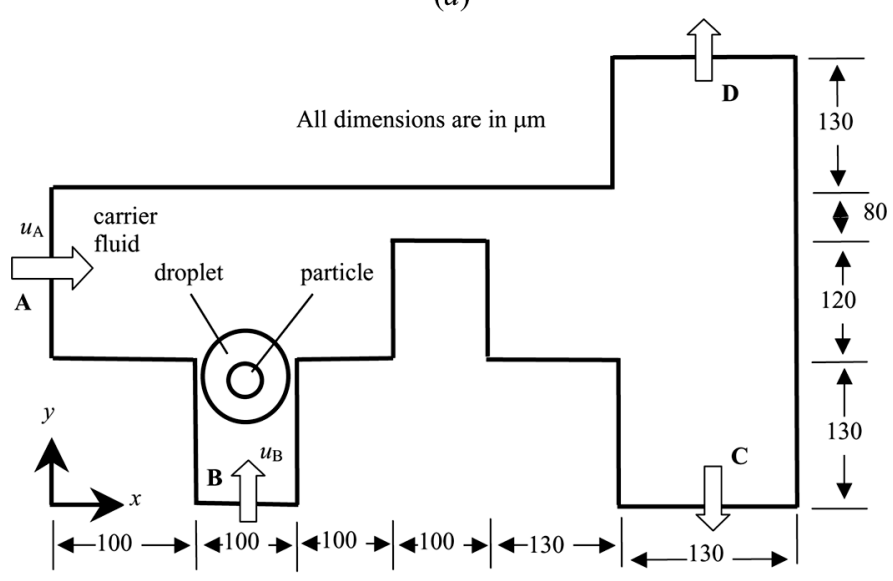

(b)

Figure 2. Schematics: $(a)$ encapsulated particle through a constriction; $(b)$ encapsulated particle through a microchannel system. 
flows into the microchannel with a uniform velocity at the inlet. The droplet is then squeezed through the constriction. Figure $2 b$ shows a particle initially encapsulated inside a circular droplet entering a microchannel system through one of the branches. The particle is carried downstream by the main carrier fluid, which enters the system at two inlets as shown in Figure $2 b$.

In both situations, the droplet, together with the particle, is carried by the pressure-driven immiscible carrier fluid. As the carrier fluid flows, it exerts a hydrodynamic force on the droplet in the form of surface stress, carrying the droplet with it. Meanwhile, the flowing fluid inside the droplet interacts with the solid particle, and an exchange of momentum occurs. This generates a hydrodynamic force on the solid particle. As a result, the solid particle undergoes both translational and rotational motions.

For the present study, only the situations where the solid particle remains encapsulated in the droplet are considered. As a result, two different interfaces, namely, the droplet-carrier fluid interface and the droplet-particle interface, are encountered. While the former is a liquid-liquid $(\mathrm{L}-\mathrm{L})$ interface, the later is a liquid-solid (L-S) interface.

For the carrier fluid, the microchannel wall is a stationary boundary but the $\mathrm{L}-\mathrm{L}$ interface is a moving boundary. For the droplet, both the $\mathrm{L}-\mathrm{L}$ and the $\mathrm{L}-\mathrm{S}$ interfaces are moving boundaries. The motion of the droplet and the encapsulated particle generates entirely new $\mathrm{L}-\mathrm{L}$ and $\mathrm{L}-\mathrm{S}$ boundaries for the flow field. Consequently, the flow field changes, which in turn affects the motion of the droplet and the particle.

\section{MATHEMATICAL FORMULATIONS}

This section of the article consists of six subsections. The first and second subsections are devoted to the representation and tracking of the $\mathrm{L}-\mathrm{L}$ and the $\mathrm{L}-\mathrm{S}$ interfaces. With these interfaces defined, the Navier-Stokes equations are then written for the whole domain, i.e., carrier fluid, droplet, and particle regions. To complete the mathematical model, the associated boundary conditions are presented. A brief description of the numerical methods employed in the discretization of the governing equations is given. Finally, the solution procedure is listed.

\subsection{L-L Interface Capturing}

The level-set method [5] with the global mass correction scheme of Yap et al. [4] is used to capture the $\mathrm{L}-\mathrm{L}$ interface. The level-set function $\xi$ is defined as the signed distance function from the $\mathrm{L}-\mathrm{L}$ interface. Obviously, the value of $\xi$ at the interface is zero. In the present study, the value of $\xi$ in the carrier fluid is assigned negative. As $\xi$ captures only the $\mathrm{L}-\mathrm{L}$ interface, the distance function $\xi$ for both the droplet and the particle regions is positive. As a result, $\xi$ can be expressed as

$$
\xi(\vec{x}, t)= \begin{cases}<0 & \text { if } \vec{x} \in \text { carrier fluid region } \\ =0 & \text { if } \vec{x} \in \mathrm{L}-\mathrm{L} \text { interface } \\ >0 & \text { if } \vec{x} \in \text { dropet or particle region }\end{cases}
$$


The evolution of the level-set function is obtained using

$$
\frac{\partial \xi}{\partial t}+u_{j} \frac{\partial \xi}{\partial x_{j}}=0
$$

To ensure that $\xi$ remains a distance function, redistancing is performed. This is achieved by solving for the "steady-state" solution of a second distance function $\psi$ given by

$$
\frac{\partial \psi}{\partial \bar{t}}=\operatorname{sign}(\xi)(1-|\nabla \psi|)
$$

The "initial" condition for Eq. (3) is $\psi(\vec{x}, 0)=\xi(\vec{x})$. Even with proper redistancing, mass might not be conserved. To overcome this, the global mass correction (GMC) procedure of Yap et al. [4] is used. The "steady-state" solution to a third distance function $\psi^{\prime}$ that ensures proper mass conservation is solved. This is written as

$$
\frac{\partial \psi^{\prime}}{\partial t^{\prime}}=\operatorname{sign}\left(\xi_{\text {ref }}\right) \frac{M_{d}-M_{c}}{M_{d}}
$$

where $M_{d}$ and $M_{c}$ are the desired mass and the most current mass of the reference phase, respectively. The term $\operatorname{sign}\left(\xi_{\text {ref }}\right)$ refers to the sign of the reference phase. The droplet is chosen as the reference phase in this article. The "steady-state" values of $\psi$ are used as the "initial" condition for Eq. (4).

\subsection{L-S Interface Tracking}

Particle motion in microchannels is modeled using a recently proposed distance function-based approach of Yap et al. [6]. The L-S interface is represented by a signed distance function $\phi$. It is defined as the normal signed distance from the $\mathrm{L}-\mathrm{S}$ interface. Obviously, at the $\mathrm{L}-\mathrm{S}$ interface, $\phi=0$. In order to differentiate the liquid region (both carrier fluid and droplet) from the particle region, $\phi$ of the fluid region is assigned a positive sign. Therefore,

$$
\phi(\vec{x}, t)= \begin{cases}<0 & \text { if } \vec{x} \in \text { particle region } \\ =0 & \text { if } \vec{x} \in \mathrm{L}-\mathrm{S} \text { interface } \\ >0 & \text { if } \vec{x} \in \text { carrier fluid or droplet region }\end{cases}
$$

Since the particle does not deform, $\phi$ can be calculated from the knowledge of the particle centroid $\vec{x}_{p}$ and orientation $\theta_{p}$. The particle centroid and orientation are determined from the motion of the particle. The translational and rotational motions of the particle are governed respectively by

$$
m_{p} \frac{d^{2} \vec{x}_{p}}{d t^{2}}=\vec{F}
$$


and

$$
I \frac{d^{2} \theta_{p}}{d t^{2}} \hat{k}=\vec{T}
$$

with $\vec{x}_{p}, d \vec{x}_{p} / d t, \theta_{p}$, and $d \theta_{p} / d t$ known at $t=0$. The resultant surface force $\vec{F}$ and torque $\vec{T}$ acting on the particle are given by

$$
\begin{gathered}
\vec{F}=\int_{\Gamma_{p}} \overline{\bar{\sigma}} \cdot \hat{n} d A \\
\vec{T}=\int_{\Gamma_{p}}\left(\vec{x}-\vec{x}_{p}\right) \times \overline{\bar{\sigma}} \cdot \hat{n} d A
\end{gathered}
$$

To facilitate calculations, Eqs. $(8 a)$ and $(8 b)$ are converted into volume integrations as

$$
\begin{gathered}
\vec{F}=\int_{V} \delta(\phi-\varepsilon) \overline{\bar{\sigma}} \cdot \hat{n} d V \\
\vec{T}=\int_{V} \delta(\phi-\varepsilon)\left(\vec{x}-\vec{x}_{p}\right) \times \overline{\bar{\sigma}} \cdot \hat{n} d V
\end{gathered}
$$

where the Dirac delta function $\delta(\phi-\varepsilon)$ is defined such that

$$
\delta(\phi-\varepsilon)= \begin{cases}\{1+\cos [\pi(\phi-\varepsilon) / \varepsilon]\} /(2 \varepsilon) & 0 \leq \phi \leq 2 \varepsilon \\ 0 & \text { otherwise }\end{cases}
$$

Further details are presented in [6] and will not be repeated here.

\subsection{Continuity and Navier-Stokes Equations for the Whole Domain}

In this article, a combined formulation with one set of governing equations to treat the three phases is employed. The carrier fluid is assumed incompressible, while the particle does not deform. The solid particle is modeled as a highly viscous fluid. With this, the "fluid" in the particle region behaves as if it is a rigid body and is therefore constrained to move with rigid body motion. The continuity and the momentum equations are

$$
\begin{gathered}
\frac{\partial \rho}{\partial t}+\nabla \cdot(\rho \vec{u})=0 \\
\frac{\partial(\rho \vec{u})}{\partial t}+\nabla \cdot(\rho \vec{u} \vec{u})=-\nabla p+\nabla \cdot\left[\mu\left(\nabla \vec{u}+\nabla \vec{u}^{T}\right)\right]-\delta\left(\vec{x}-\vec{x}_{\Gamma_{L}}\right) \sigma \kappa \hat{n}
\end{gathered}
$$

In Eq. (11), the surface tension term is modeled using the continuum surface force model [8]. Equations (10) and (11) apply to the whole domain. As such, $\rho$ and $\mu$ 
represent the density and viscosity appropriate for the phase occupying the particular spatial location at a given instant of time. These properties are expressed as

$$
\alpha(\phi, \xi)= \begin{cases}\alpha_{p} & \phi<0 \\ (1-H) \alpha_{c}+H \alpha_{d} & \phi>0\end{cases}
$$

where the subscripts $p, c$, and $d$ stand for particle, carrier fluid, and droplet, respectively. The symbol $\alpha$ can be either density or viscosity. The following definition of the Heaviside function $H$ is used [7]:

$$
H= \begin{cases}0 & \xi<-\varepsilon \\ \frac{\xi+\varepsilon}{2 \varepsilon}+\frac{1}{2 \pi} \sin \left(\frac{\pi \xi}{\varepsilon}\right) & |\xi| \leq \varepsilon \\ 1 & \xi>\varepsilon\end{cases}
$$

\subsection{Boundary Conditions}

The axial velocities at the inlets are set to a uniform value. The transverse velocities at these sections are assumed to be zero. No-slip condition is imposed on the microchannel walls. At the outlets, the axial gradients of the transverse velocities are set to zero. The axial velocities are calculated to ensure mass conservation.

The L-S interface serves as the moving boundary for the fluid. The condition at the moving $\mathrm{L}-\mathrm{S}$ boundary is imposed in the following manner. An inner layer of the solid particle, defined as $-\varepsilon \leq \phi \leq 0$, is set to have rigid body motion. The velocity in this region is specified as

$$
\vec{u}=\frac{d \vec{x}_{p}}{d t}+\frac{d \theta_{p}}{d t} \hat{k} \times\left(\vec{x}-\vec{x}_{p}\right)
$$

where the first and the second terms on the right represent the translational and the rotational motions of the particle, respectively. The velocity in the remaining region of the solid body defined by $\phi<-\varepsilon$ is forced to be zero. The setting of the velocity in these two regions of the solid body is achieved using the internal control-volume approach of Patankar [9]. Strictly speaking, this two-region treatment inside the solid is not needed for this situation, where the particle undergoes rigid body translation and rotation and there are no additional forces (such as electrophoretic force) which result in slip velocity at the particle-fluid interface. This two-region formulation is introduced in anticipation of slip velocity at the particle-fluid surface, as in the case of electrophoretic motion of a solid particle in an aqueous solution. This situation is not discussed here.

\subsection{Numerical Method}

The flow field and the level-set functions are solved using the finite-volume method of Patankar [9]. A staggered grid is used in this article. The scalar variables are stored at the centers of the control volumes, while the velocities are located at the control-volume faces. In this article, the power-law scheme is used to model the combined convection-diffusion effect in the momentum equations. The SIMPLER 
algorithm is used to resolve the velocity-pressure coupling. The CLAM scheme [10], which is of second-order accuracy, is used to model the convection of the level-set equations. The fully implicit scheme is used to discretize the transient term.

\subsection{Solution Procedure}

The solution procedure can be summarized as follows.

1. Initialize $\vec{u}$, $\xi$, and $\phi$ for $t_{n}=0(n=0)$.

2. Set $t_{n+1}=t_{n}+\Delta t$.

3. Calculate $\rho$ and $\mu$ from Eq. (12).

4. Solve for $\vec{u}$ and $p$ from Eqs. (10) and (11).

5. Solve for $\xi$ [Eq. (2)], perform redistancing [Eq. (3)] and mass correction [Eq. (4)].

6. Calculate $\vec{F}$ and $\vec{T}$ from Eqs. $(9 a)$ and $(9 b)$.

7. Calculate $\vec{x}_{p}$ [Eq. (6)] and $\theta_{p}$ [Eq. (7)] and reconstruct $\phi$.

8. Repeat steps (3)-(7) until the solution converges.

9. Repeat steps (2)-(8) for all time steps.

\section{RESULTS AND DISCUSSION}

\subsection{Validation}

The $\mathrm{L}-\mathrm{L}$-interface-capturing procedure has been validated by Yap et al. [4] against the volume-of-fluid (VOF) method for droplet flowing in double-bend, straight, and constricted microchannels. The $\mathrm{L}-\mathrm{S}$ interface tracking procedure was validated by Yap et al. [6] via flow around stationary, forced, and freely rotating cylinders. In addition, the trajectories of a settling cylinder were also computed and validated against existing results.

\subsection{Encapsulated Particle through a Constricted Microchannel}

The motion of a circular particle encased in a droplet is considered first. A particle of diameter $d_{p}=40 \mu \mathrm{m}$ is initially suspended at $\left(\left(x_{p}, y_{p}\right)=(150,99.5) \mu \mathrm{m}\right)$. The density of the particle is set to $\rho_{p}=2,700 \mathrm{~kg} / \mathrm{m}^{3}$. The particle is encased by a droplet of diameter $d_{d}=170 \mu \mathrm{m}$ and located at $\left(x_{d}, y_{d}\right)=(150,99.5)$. The density $\rho_{d}$ and viscosity $\mu_{d}$ of the droplet are set to $1,000 \mathrm{~kg} / \mathrm{m}^{3}$ and $0.001 \mathrm{Ns} / \mathrm{m}^{2}$, respectively. The density $\rho_{f}$ and viscosity $\mu_{f}$ of the carrier fluid are $800 \mathrm{~kg} / \mathrm{m}^{3}$ and $0.01 \mathrm{Ns} / \mathrm{m}^{2}$, respectively. Surface tension $\sigma$ between droplet and the carrier fluid is set to $10^{-4} \mathrm{~N} / \mathrm{m}$. The inlet velocity of the carrier fluid is $2,000 \mu \mathrm{m} / \mathrm{s}$. These parameters are retained in all the subsequent cases unless stated otherwise.

The evolution of the $\mathrm{L}-\mathrm{L}$ interface and the motion of the particle are shown in Figure 3. Solutions were obtained using uniform meshes of $173 \times 41$ control volumes (CVs) with $\Delta t=4.0 \times 10^{-5} \mathrm{~s}$ and $346 \times 82 \mathrm{CVs}$ with $\Delta t=2.0 \times 10^{-5} \mathrm{~s}$. Neither the $\mathrm{L}-\mathrm{L}$ nor the $\mathrm{L}-\mathrm{S}$ interfaces show any noticeable differences for these mesh sizes. Therefore, a mesh of $173 \times 41 \mathrm{CVs}$ with $\Delta t=4.0 \times 10^{-5} \mathrm{~s}$ is sufficient to achieve spatially and temporally independent solutions. The droplet deforms significantly as it squeezes through the constriction. Surface tension minimizes the droplet surface 


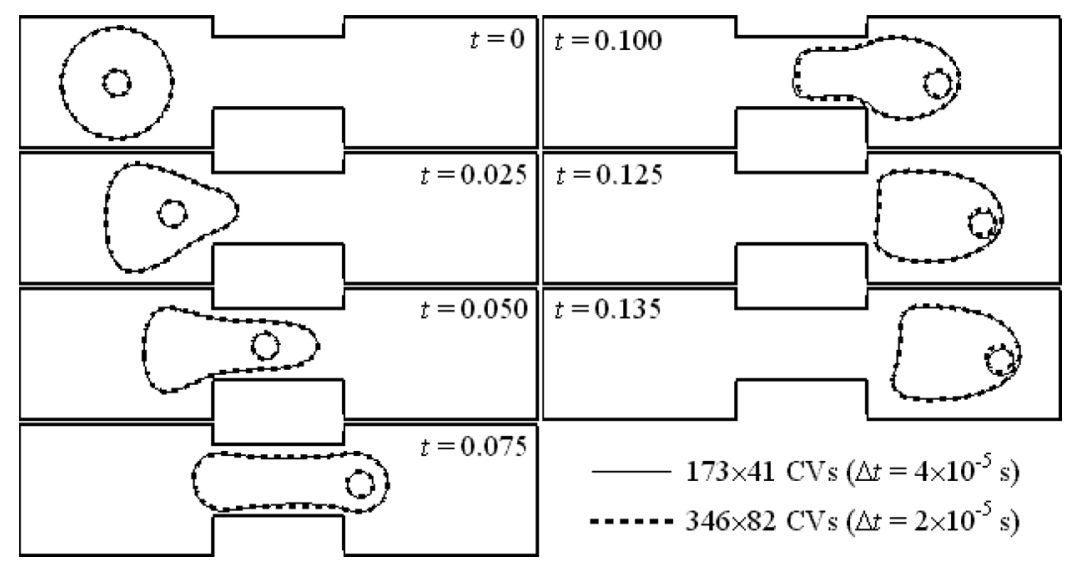

Figure 3. Flow of a $40-\mu \mathrm{m}$ particle-encapsulated droplet.

area. The particle, which is initially located at the center of the droplet, is increasingly pushed downstream toward the "frontal portion" of the droplet. As the droplet emerges from the constriction, the droplet becomes more "bullet-like."

For this computation, GMC is employed. The mass errors with and without GMC are shown in Figure 4. Without GMC, there is around 12\% mass loss for the droplet at the end of the computation. As seen in Figure 4, the mass of droplet fluid is well conserved (on the order of $10^{-15}$ ) with GMC.

The effect of the carrier fluid viscosity is shown in Figure 5. Three different viscosities, namely, $0.001,0.005$, and $0.010 \mathrm{Ns} / \mathrm{m}^{2}$, are considered. The droplet and the particle at $t=0,0.025,0.050,0.075,0.100,0.125$, and $0.150 \mathrm{~s}$ are shown alternatively as solid or dashed line. As $\mu_{c}$ increases, the boundary-layer thickness of the carrier fluid increases. This is especially evident in the constriction. The droplet is squeezed in such a way that the $\mathrm{L}-\mathrm{L}$ interface is farther away from the wall. The cross-sectional area available for the droplet to flow decreases. Therefore, the droplet flows faster. This generates a larger hydrodynamic force on the encapsulated particle and propels it farther downstream with a higher velocity. When the droplet

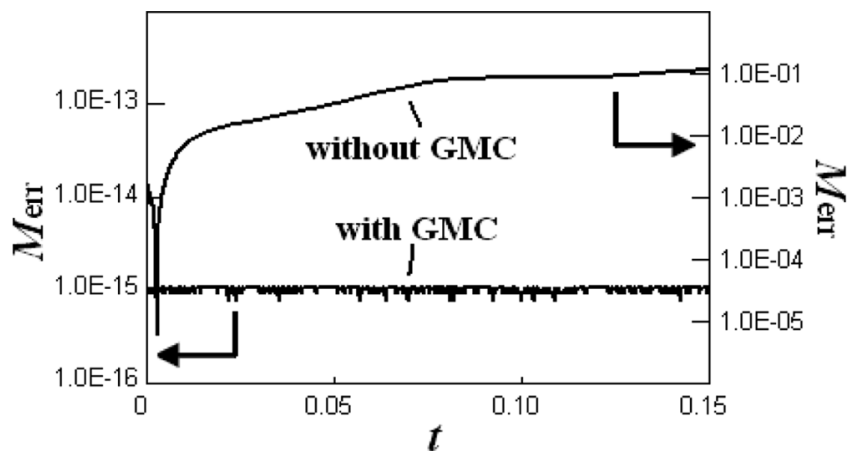

Figure 4. Mass error of the reference fluid (fluid 1) with and without GMC. 


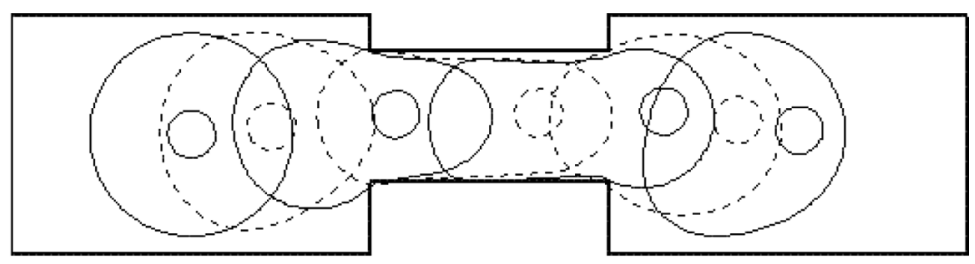

(a) $\mu_{c}=0.001 \mathrm{Ns} / \mathrm{m}^{2}$

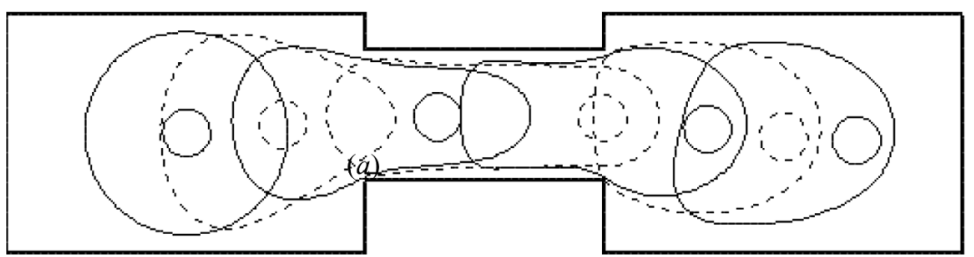

(b) $\mu_{c}=0.005 \mathrm{Ns} / \mathrm{m}^{2}$

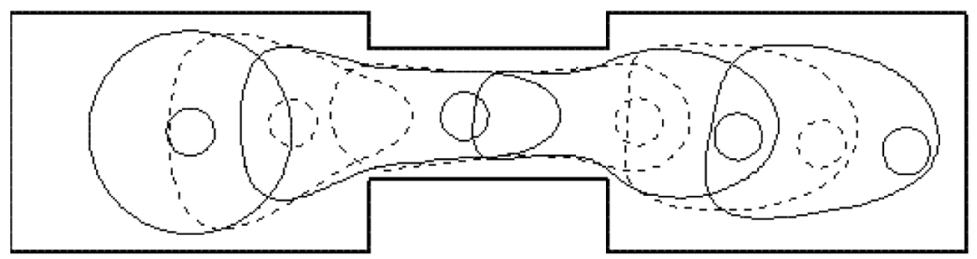

(c) $\mu_{c}=0.010 \mathrm{Ns} / \mathrm{m}^{2}$

Figure 5. Effect of viscosity ratio.

emerges from the constriction, surface tension minimizes the $\mathrm{L}-\mathrm{L}$ interfacial area by rounding out the frontal portion of the droplet. However, the effect of the boundary layer of the carrier fluid remains significant. It pushes the portions of the $\mathrm{L}-\mathrm{L}$ interface near the wall to the center of the channel, compressing the droplet into a more "bullet-like" shape for larger carrier fluid viscosity.

The evolution of the particle-encapsulated droplet with different surface tensions is depicted in Figure 6. The surface tension $\sigma$ is set to $0.5 \times 10^{-4}$, $1.0 \times 10^{-4}$, or $2.5 \times 10^{-4} \mathrm{~N} / \mathrm{m}$. The droplet and the particle at $t=0,0.025,0.050$, $0.075,0.100,0.125$, and $0.150 \mathrm{~s}$ are shown. The surface tension $\sigma$ minimizes the $\mathrm{L}-\mathrm{L}$ interfacial area. For a circular droplet, it maintains the circular shape of the droplet as the $\mathrm{L}-\mathrm{L}$ interfacial area is minimal. Therefore, $\sigma$ acts to resist any stretching of the droplet by the flow field. Larger $\sigma$ implies more resistance to deformation and thus smaller stretching. When the droplet flows into the constriction, severe stretching occurs. For the case of larger $\sigma$, stretching is smaller, with the frontal and rear portion of the droplet rounded out more. This is depicted lucidly in the $\mathrm{L}-\mathrm{L}$ interface at $t=0.050$ and $0.100 \mathrm{~s}$. The droplet flows more slowly given an increase in cross-sectional area for it to flow. A smaller hydrodynamic force acts on the encapsulated particle. Therefore, the particle moves more slowly. After the droplet emerges from the constriction, $\sigma$ tries to restore the circular shape of the droplet, lowering the velocity of the $\mathrm{L}-\mathrm{L}$ interface in the frontal portion of the droplet. This is because the surface tension force which is tangential to the interface has a 


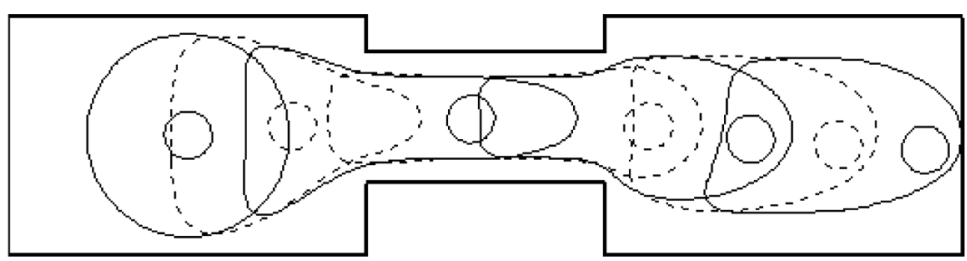

(a) $\sigma=0.5 \times 10^{-4} \mathrm{~N} / \mathrm{m}$

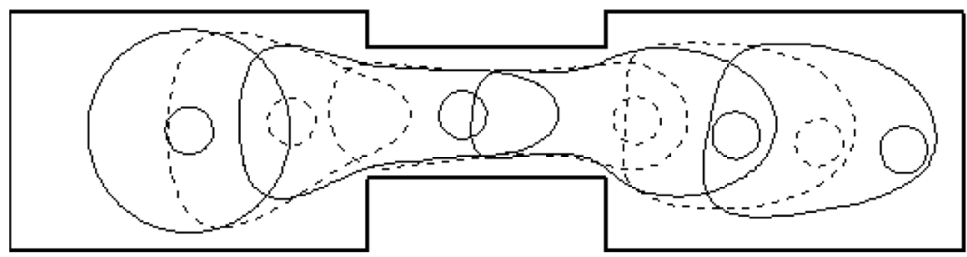

(b) $\sigma=1.0 \times 10^{-4} \mathrm{~N} / \mathrm{m}$

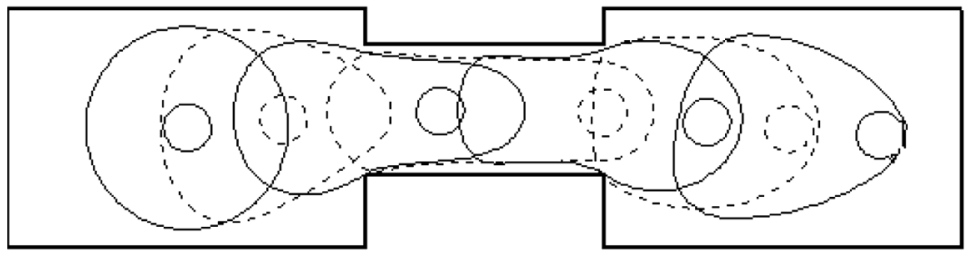

(c) $\sigma=2.5 \times 10^{-4} \mathrm{~N} / \mathrm{m}$

Figure 6. Effect of surface tension.

resultant backward component. Such a slowdown in the $\mathrm{L}-\mathrm{L}$ interface movement causes the particle to break out from the encapsulation for the case of $\sigma=2.5 \times 10^{-5} \mathrm{~N} / \mathrm{m}$.

The effect of different particle densities is now investigated. Three different particle densities, namely, 2,700, 6,000, and $8,960 \mathrm{~kg} / \mathrm{m}^{3}$, are considered. When the particle is impulsively acted upon by the hydrodynamic force, the inertia effect is found to be significant only in a very short period of time. Figure 7 shows the variation of the axial velocity over this period of time, beyond which no obvious dependence of the axial velocity on the particle density is observed. Since the particles are of the same size, a larger particle density implies a larger mass. The inertia is larger and therefore it has more resistance to motion. The denser particle experiences a smaller acceleration and thus moves with a smaller velocity. It takes a slightly longer time to attain the terminal axial velocity. The distance covered in this time interval is only about $20-30 \mu \mathrm{m}$, which is relatively small compared to the total distance the particle travels downstream (around $700 \mu \mathrm{m}$ ). This indicates that the effect of particle density on the overall movement of the particle is negligible. Therefore, no obvious effect on the evolution of the $\mathrm{L}-\mathrm{L}$ interface is observed (Figure 8).

\subsection{Flow of Particle-Encapsulated Droplets in a Microchannel System}

Figure $2 b$ shows a particle-encapsulated droplet in a microchannel system. The $80-\mu \mathrm{m}$-diameter droplet is initially suspended at $(150,117)$. The density and viscosity 


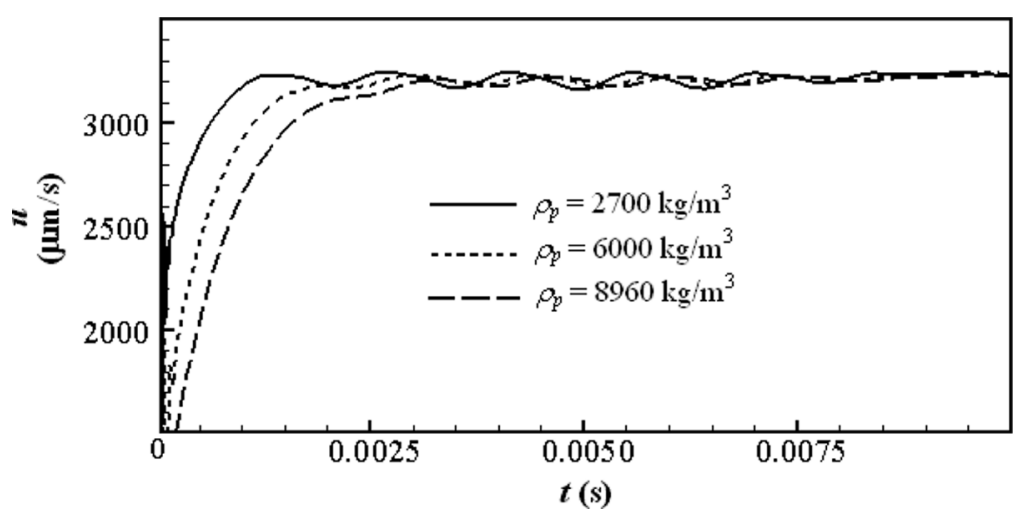

Figure 7. Effect of particle density on the "axial" velocity of the particle.

of the droplet are $\rho_{d}=2,000 \mathrm{~kg} / \mathrm{m}^{3}$ and $\mu_{d}=0.002 \mathrm{Ns} / \mathrm{m}^{2}$, respectively. A circular solid particle of diameter $d_{p}=30 \mu \mathrm{m}$ and density $\rho_{d}=8,960 \mathrm{~kg} / \mathrm{m}^{3}$ is encased in the droplet. At both inlets $\mathrm{A}$ and $\mathrm{B}$, the same carrier fluid flows into the microchannel at $1,000 \mu \mathrm{m} / \mathrm{s}$ and $4,000 \mu \mathrm{m} / \mathrm{s}$, respectively. Its density and viscosity are $\rho_{c}=1,000 \mathrm{~kg} / \mathrm{m}^{3}$ and $\mu_{c}=0.001 \mathrm{Ns} / \mathrm{m}^{2}$, respectively. The surface tension is set to $\sigma=1 \times 10^{-5} \mathrm{~N} / \mathrm{m}$ for demonstration purposes.

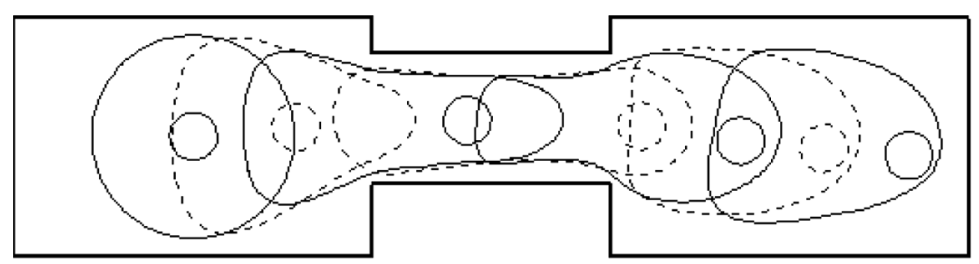

(a) $\rho_{p}=2700 \mathrm{~kg} / \mathrm{m}^{3}$

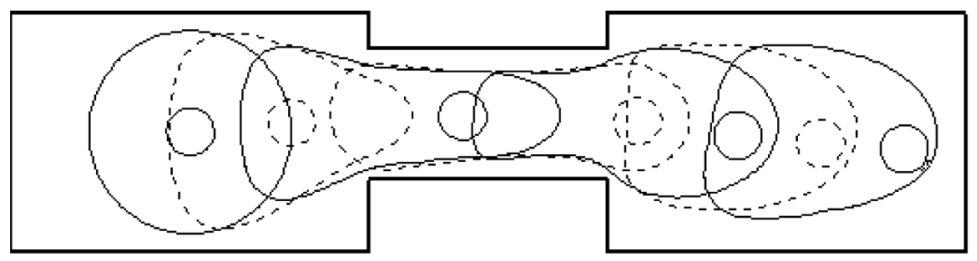

(b) $\rho_{p}=6000 \mathrm{~kg} / \mathrm{m}^{3}$

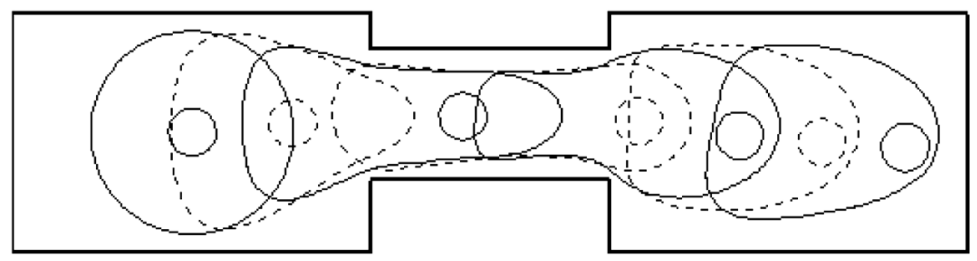

(c) $\rho_{p}=8960 \mathrm{~kg} / \mathrm{m}^{3}$

Figure 8. Effect of particle density. 


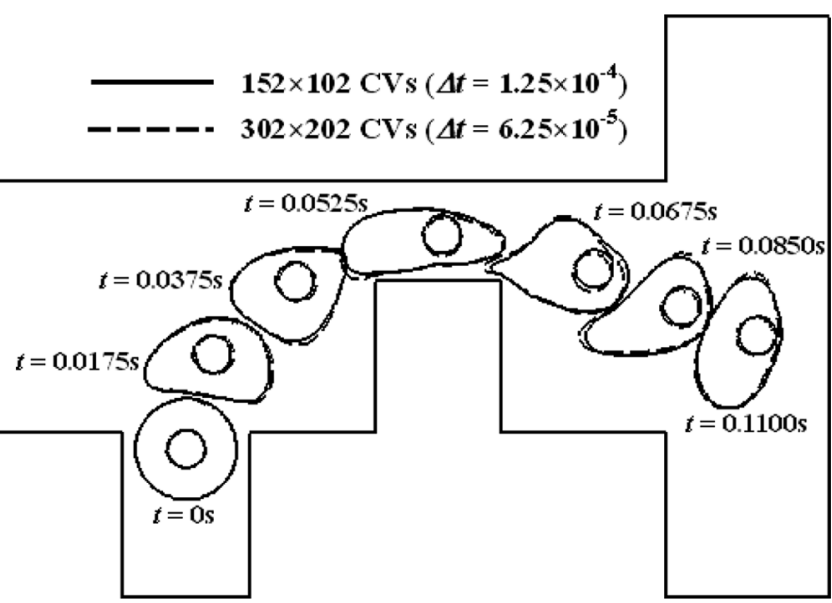

Figure 9. Evolution of a particle-encapsulation droplet in a microchannel system.

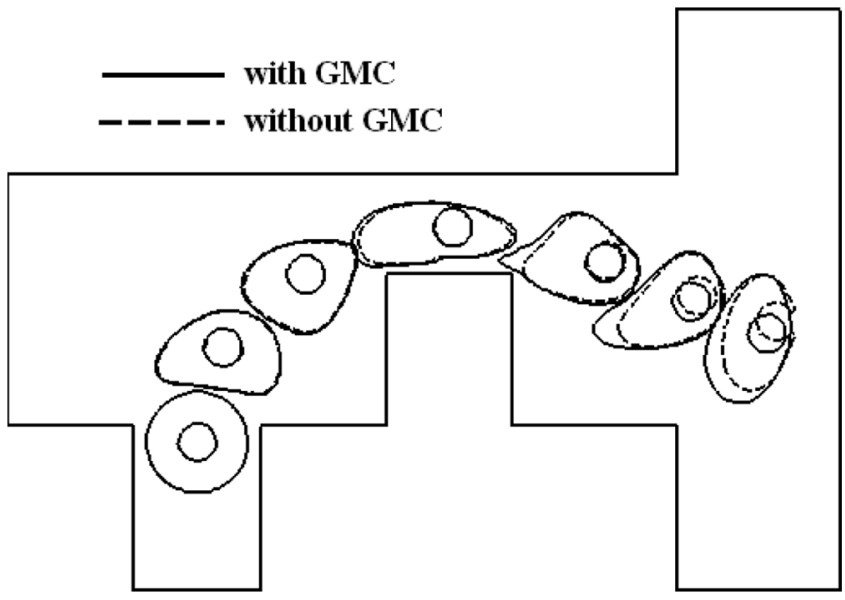

Figure 10. Evolution of the particle-encapsulated droplet with and without GMC.

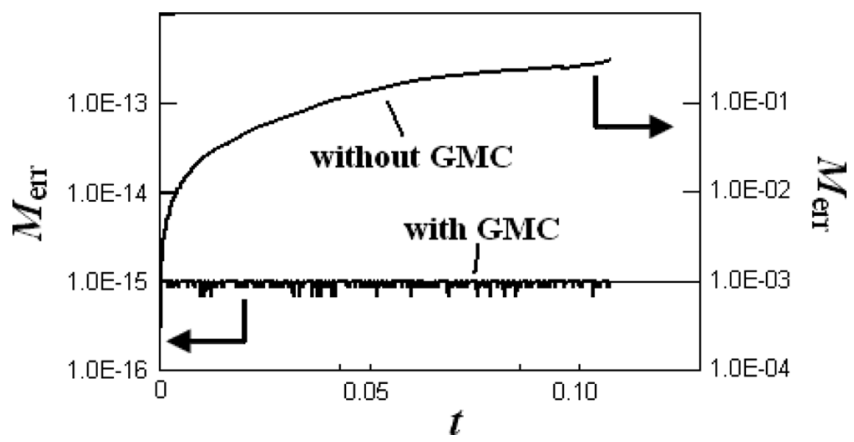

Figure 11. Mass error of the reference fluid (fluid 1) with and without GMC. 
Figure 9 shows the evolutions of the $\mathrm{L}-\mathrm{L}$ and $\mathrm{L}-\mathrm{S}$ interfaces obtained using two spatial and temporal grids. It is seen that a mesh of $152 \times 102 \mathrm{CVs}$ with $\Delta t=1.25 \times 10^{-4} \mathrm{~s}$ is sufficient to capture all the essence of the interfaces. As a result, these spatial and temporal grids are used for all the remaining computations reported in this article. There are two situations where the particle is prone to break out from the droplet. These are (1) as the droplet is flowing through the constriction and (2) as the droplet is making a turn. For (2), the inertia of the particle is larger than the droplet. A larger force is required for the particle to make the same turn. Therefore, it does not follow the droplet trajectory exactly and tends to break out from the droplet. It should be pointed out that as the droplet turns into branch $\mathrm{C}$,
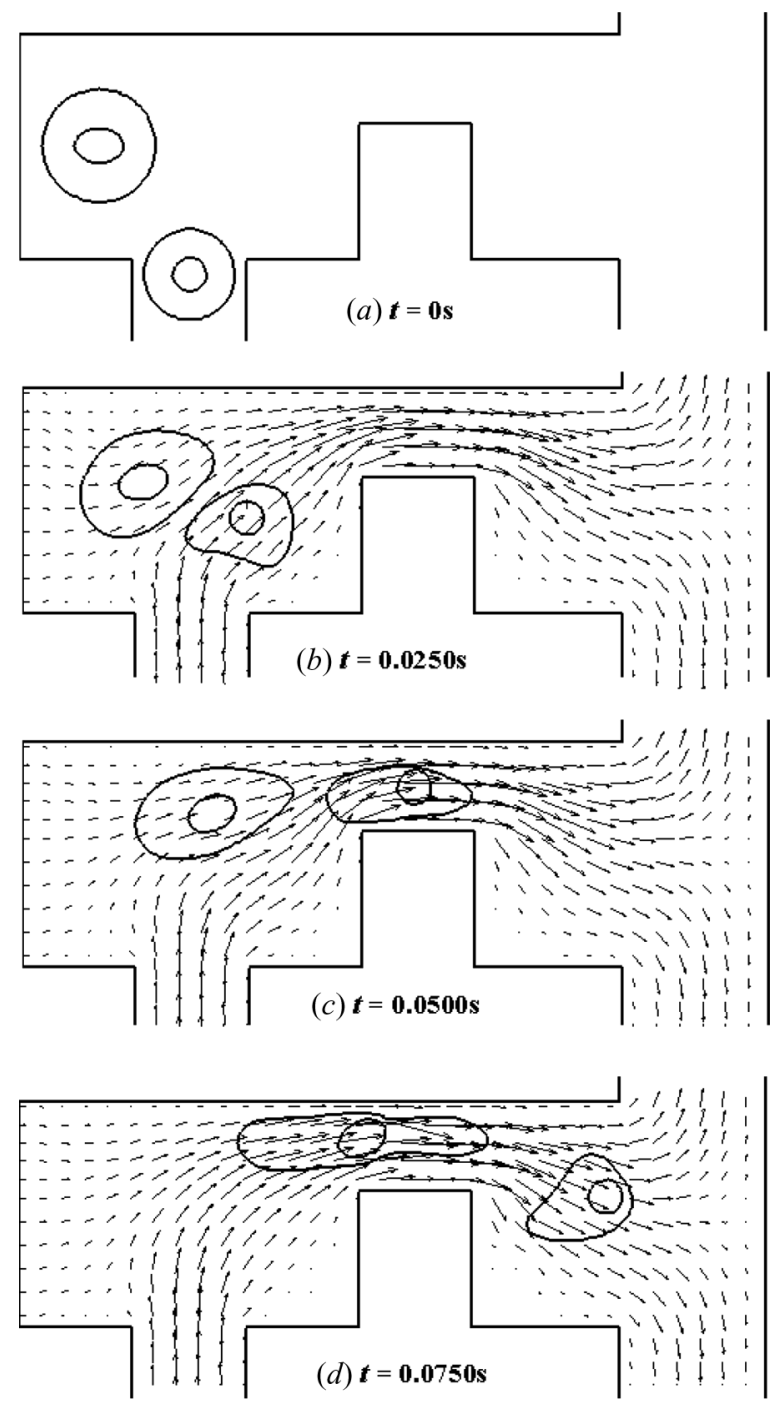

Figure 12. Evolution of two particle-encapsulation droplets in a microchannel system. 

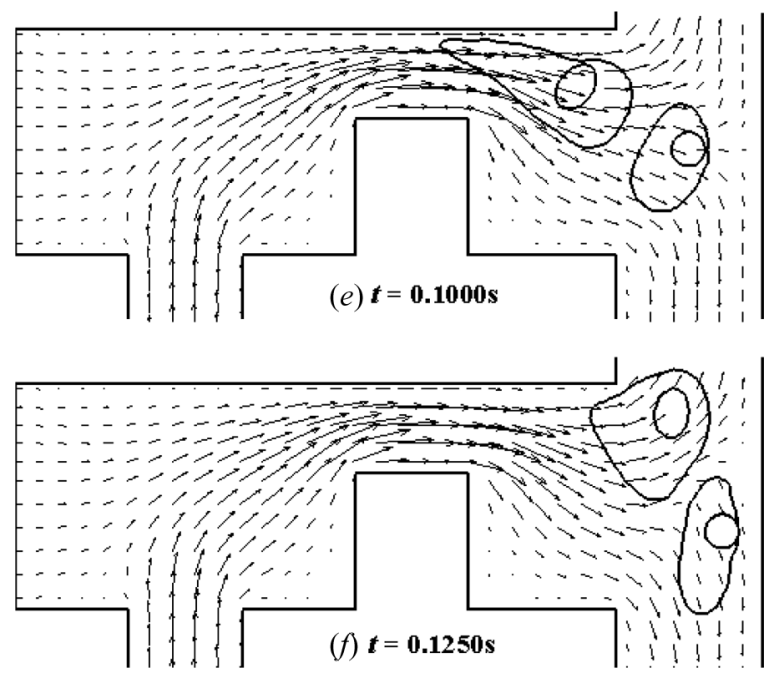

Figure 12. Continued.

the encapsulated particle breaks out from the droplet. Computation is stopped at $t=$ $0.1100 \mathrm{~s}$ as postbreakout flow is not the present interest. Such a computation involves a three-phase-contact-line problem, which is not addressed in the present study.

The evolution of the particle-encapsulated droplet with and without GMC is shown in Figure 10. Different $\mathrm{L}-\mathrm{L}$ and $\mathrm{L}-\mathrm{S}$ interfaces are predicted. The droplet for the case without GMC is increasingly smaller than that with GMC after it emerges from the constriction. The mass error for the reference fluid (droplet fluid) is shown in Figure 11. Without GMC, the mass loss of the particle-encapsulated droplet is increasing over time. Substantial mass, up to almost $30 \%$, is lost at the end of the computation for the droplet. With acute mass loss, even the L-S interface can be affected as the flow field changes due to a "smaller" droplet. Again, GMC conserves the reference fluid mass well.

The application of the present model for the case of two particle-encapsulated droplets is now presented. A second droplet is added. This droplet of diameter $100 \mu \mathrm{m}$ is located at $(70,306)$. It encases an elliptical particle with major and minor axes of $44 \mu \mathrm{m}$ and $30 \mu \mathrm{m}$, respectively. The particle is initially located at the center of the droplet. Figure 12 shows the evolution of the $\mathrm{L}-\mathrm{L}$ and $\mathrm{L}-\mathrm{S}$ interfaces. One in every five velocity vectors is shown, to avoid overcrowding the figure. The elliptical particle-encapsulated droplet is trailing the circular particle-encapsulated droplet given the smaller driving flow at inlet A. The elliptical particle undergoes obvious rotational motion. Examination of the velocity field suggests that the circular and elliptical particles are driven to outlets $\mathrm{C}$ and $\mathrm{D}$, respectively. Computation is stopped at $t=0.1250 \mathrm{~s}$, when the circular particle breaks out from the droplet.

\section{CONCLUDING REMARKS}

A fixed-grid method has been presented to model the motion of particleencapsulated droplets carried by a pressure-driven immiscible carrier fluid in 
microchannels. With the solid particle modeled as a highly viscous fluid that is constrained to move with rigid body motion, the three phases are treated together using a combined formulation with only one set of governing equations. The level-set function and a distance function are used to represent and capture the droplet-carrier fluid and droplet-particle interfaces. The governing equations are solved numerically using a finite-volume method on a fixed Cartesian grid. Using the present model, the effects of carrier fluid viscosity, surface tension, and particle density have been investigated for the flow of a particle-encapsulated droplet through a constriction. Flows

of a single and two particle-encapsulated droplets in a microchannel system have been demonstrated.

\section{REFERENCES}

1. J. Atencia and D. J. Beebe, Controlled Microfluidic Interfaces, Nature, vol. 437, pp. 648655, 2005.

2. J. S. Fisher and A. P. Lee, Cell Encapsulation on a Microfluidic Platform, MicroTAS 2004, Malmo, Sweden, September 26-30, 2004.

3. M. He, J. S. Edgar, G. D. M. Jeffries, R. M. Lorenz, J. P. Shelby, and D. T. Chiu, Selective Encapsulation of Single Cells and Subcellular Organelles into Picoliter and Femtoliter-Volume Droplets, Anal. Chem, vol. 77, pp. 1539-1544, 2005.

4. Y. F. Yap, J. C. Chai, T. N. Wong, K. C. Toh, and H. Y. Zhang, A Global Mass Correction Scheme for the Level-Set Method, Numer. Heat Transfer B, vol. 50, pp. 455-472, 2006.

5. S. Osher and J. A. Sethian, Fronts Propagating with Curvature-Dependent Speed: Algorithms Based on Hamilton-Jacobi Formulations, J. Comput. Phys., vol. 79, pp. 12-49, 1988.

6. Y. F. Yap, J. C. Chai, T. N. Wong, K. C. Nguyen, K. C. Toh, and H. Y. Zhang, Particle Transport in Microchannels, Numer. Heat Transfer B, vol. 51, pp. 141-157, 2007.

7. Y. C. Chang, T. Y. Hou, B. Merriman, and S. Osher, A Level Set Formulation of Eulerian Interface Capturing Methods for Incompressible Fluid Flows, J. Comput. Phys., vol. 124, pp. 449-464, 1996.

8. J. U. Brackbill, D. B. Kothe, and C. Zemach, A Continuum Method for Modelling Surface Tension, J. Comput. Phys., vol. 100, pp. 335-354, 1992.

9. S. V. Patankar, Numerical Heat Transfer and Fluid Flow, Hemisphere, New York, 1980.

10. B. Van Leer, Towards the Ultimate Conservative Difference Scheme. II. Monotonicity and Conservation Combined in a Second Order Scheme, J. Comput. Phys., vol. 14, pp. 361-370, 1974. 